\title{
Immunocompetence in Hydra: Epithelial Cells Recognize Self-Nonself and React Against It
}

\author{
THOMAS C.G. BOSCH AND CHARLES N. DAVID \\ Zoologisches Institut der Universität München, D-8000 München 2, \\ Federal Republic of Germany
}

\begin{abstract}
The evolution of effective immunologic defense mechanisms in multicellular organisms involves the ability of host cells to distinguish betweeen "self" and "nonself" and to react appropriately to eliminate foreign tissue. By producing interspecies grafts we have obtained evidence that immunorecognition followed by incompatibility reactions occur in Hydra. Our results demonstrate that epithelial cells of Hydra recognize and phagocytose foreign hydra cells, indicating that they are the effector cells in the incompatibility reactions. This observation is consistent with the idea that immunocompetence appeared early in the evolution of multicellular organisms.
\end{abstract}

The study of immune reactions in primitive invertebrates is important from a phylogenetic point of view. It may also contribute to our understanding of the complex immunology of higher animals since the basic principles are probably similar. In order to compare invertebrate and vertebrate phyla the term "immunity" should be used in a broad sense. An animal may be said to possess immunity if it is capable of recognizing and protecting against nonself (Lackie, '80). Involvement of specific memory in primitive immune responses may or may not occur and can be viewed as an evolving characteristic (Hildemann, '74; Lackie, '80; Coombe et al., '84).

There is now well-documented evidence of immunocompetence in a variety of multicellular invertebrates ranging from sponges and coelenterates to echinoderms and protochordates (for review see Hildemann, "79).In colonial hydrozoans and anthozoans immunocompetence characterized by specific reactivity to nonself and subsequent cytotoxic reaction is common at the level of colony specificity (Theodor, '70; Francis, '73; Hildemann, '74; Buehrer and Tardent, '80; Buss et al., '84). Interclonal histoincompatibilities under predictable genetic control have been described in the colonial hydroid Hydractinia (Hauenschild, '54, '56; Müller, '64; Buss et al., '84). More recently, autoreactivity and self-tolerance were found to occur in Hydractinia (Buss et al., '85). A specific memory component has been demonstrated in an anthozoan coral (Hildemann et al., '77).
The underlying mechanisms, which are responsible for the incompatibility reactions as well as the cellular interactions at the site of contact, are not well understood in coelenterates. In particular, little is known about the nature of the effector cells involved in histoincompatibility and cytotoxic immune reactions in coelenterates (Lackie, '80). However, in one organism, Hydractinia echinata, Buss et al., ('84) have now shown that histoincompatibility reactions are due to accumulation and discharge of specialized nematocytes at the site of contact.

To examine cellular immune specificity in the freshwater polyp Hydra we have investigated the behavior of interspecies grafts. Using maceration techniques (David, '73; Bode et al., '73) we have identified epithelial cells as the effector cells in the immunological response.

\section{MATERIALS AND METHODS}

Strains

$H$. attenuata has been cultivated in the laboratory since 1966 . The $H$. oligactis strain was obtained by Prof. P. Tardent, Zürich, in 1982.

\section{Culture condition and maceration technique}

The methods have been reported elsewhere (Bosch and David, '84).

\section{Histological methods}

To identify and count phagocytic vacuoles maceration preparations were stained by the Feulgen method as described previously 

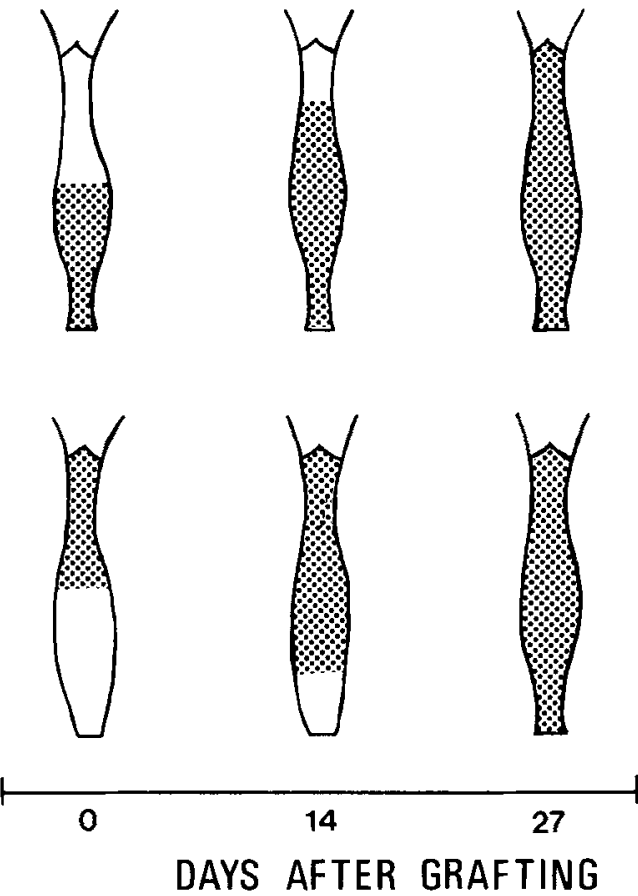

Fig. 1. Schematic drawing illustrating tissue displacement by $H$. oligactis in att/oli heterografts. $H$. attenuata (clear), H. oligactis (stippled).

(Bosch and David, '84). Developing nematoblasts in the body column were identified by staining whole mounts with lead nitratethioacetic acid (David and Challoner, '74).

\section{${ }^{3}$ H-thymidine labelling}

$\mathrm{Me}-{ }^{3} \mathrm{H}$-thymidine (specific activity $44 \mathrm{Ci} /$ $\mathrm{ml}$; Amersham Buchler, Braunschweig) was injected into the gastric cavity at a concentration of $50-100 \mu \mathrm{Ci} / \mathrm{ml}$ (David and Campbell, '72). Continuously labeling was achieved by repeated injections at 12-hour intervals.

\section{Transplantation procedure}

Heterografts were produced by stringing hydra halves onto a nylon fishline (Rubin and Bode, '82). Sleeves of polyethylene tubing were then strung onto both ends of the fishline to maintain the cut surfaces in close contact. After 1-2 hours the grafts were taken off the fishline. The grafts were inspected daily for position of the graft junction.

\section{Nematocyst analysis}

To examine the nematocyst composition in the tentacles three to five tentacles were cut off each heterograft, transferred to a micro- scope slide, and gently flattened under a coverslip. The nematocysts were analyzed by using interference microscopy. Measurements of the lengths of desmonemes were done by using a ocular micrometer.

\section{RESULTS}

Stability of interspecies grafts

Grafting success depends on the species combination and decreases as species diversity increases (Bibb and Campbell, '73). In agreement with previous reports (Campbell and Bibb, '70) investigations in our laboratory indicate that most interspecies combinations are not permanently stable. However, one pair of species, $H$. attenuata and $H$. oligactis, appears to tolerate parabiosis.

To test whether permanently stable heterografts could be produced distal halves of $H$. oligactis were grafted to proximal halves of $H$. attenuata and vice versa. Both species can be recognized due to differrent carotenoid pigments in the endoderm. $H$. attenuata is pink-orange and $H$. oligactis is yellow-orange in color (Wanek and Campbell, '82). Within 24 hours the graft junction identified by the different pigmentation was perfectly healed in the $455 \mathrm{H}$. attenuata/H. oligactis (att/oli) heterografts made thus far.

When culturing these heterografts we observed in all cases continuous tissue displacement of $H$. attenuata from the heterograft. Complete displacement required several weeks. Displacement was observed regardless of whether $H$. attenuata was the apical or the basal partner in the heterograft (Fig. 1).

\section{Cell cycle length during parabiosis}

To determine if displacement involved growth inhibition of $H$. attenuata we measured the epithelial cell cycle length of both species during parabiosis by continuous labeling with ${ }^{3} \mathrm{H}$-thymidine. Grafts were cultivated for 7 days and then "continuously" labeled with ${ }^{3} \mathrm{H}$-thymidine. After varying periods of labeling heterografts were separated at the boundary between the two partners and the labeling index of epithelial cells in each partner determined by maceration and autoradiography. The results indicate that $90 \%$ of the epithelial cells of both partners are labeled after 3 days (Fig. 2). From the labeling kinetics an epithelial cell cycle length of 3.8 days was calculated for both partners in the heterografts. In addition the 


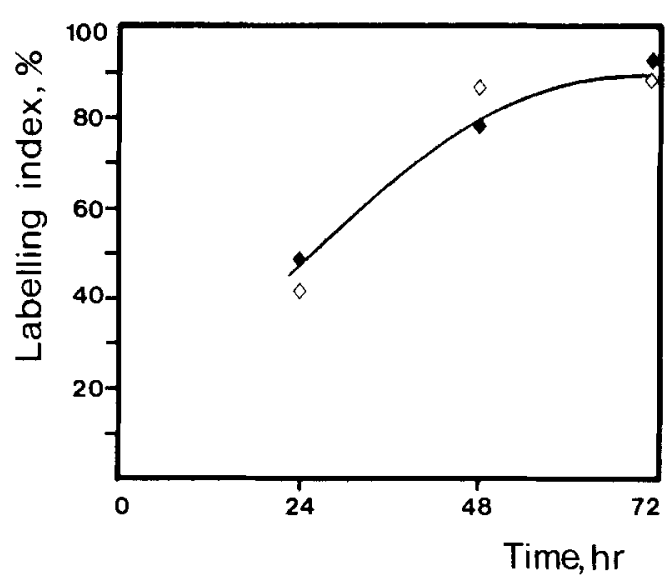

Fig. 2. ${ }^{3} \mathrm{H}$-thymidine-labeling of epithelial cells in att oli $i$ heterografts. Heterografts were cultivated for 7 days. Then the heterografts were "continuously" labeled with ${ }^{3} \mathrm{H}$-thymidine for 3 days (see Materials and Methods). Each day ten heterografts were separated at the boundary between the two partners and the labeling index in each partner was determined by maceration and autoradiography. $H$. attenuata (solid diamond), $H$, oligactis (open diamond).

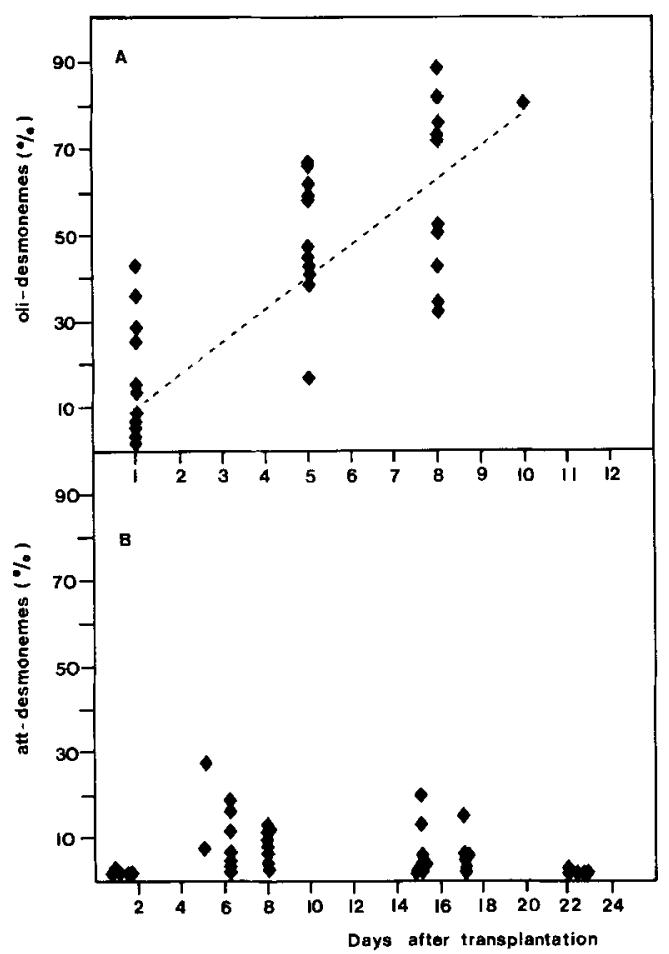

Fig. 3. A. Displacement of $H$. attenuata desmonemes in tentacles of att/oli heterografts. At each time point two to four tentacles were analyzed. After each analysis all tentacles were removed to permit regeneration of new tentacles. Thus the nematocyt content of tentacles interstitial cell population of both partners was completely labeled by this procedure (data not shown). Thus, displacement is not due to different cell cycle lengths of the two species during parabiosis.

\section{Fate of the interstitial cell lineage in heterografts}

To study the fate of cells of the interstitial cell lineage in heterografts we investigated the behavior of nematocytes in the tentacles of interspecies grafts. Desmonemes of $H$. oligactis and $H$. attenuata differ significantly in the length of their capsules (Lee and Campbell, '79). Thus it is possible to follow the fate of this cell population in the tentacles of heterografts. The results in Figure 3A indicate that $H$. oligactis desmonemes displace $H$. attenuata desmonemes at roughly the same rate as tissue displacement occurs. After 10 days about $80 \%$ of the desmonemes in tentacles of att/oli heterografts are of the $H$. oligactis type. In oliatt heterografts, where the tentacle cells are of the $H$. oligactis type, only few $H$. attenuata desmonemes were found (Fig. 3B) even at early times when half the animal consists of $H$. attenuata tissue.

To test whether displacement depended on the continued presence of $H$. oligactis tissue, att/oli heterografts were separated after 10 days (when most desmonemes were of the $H$. oligactis type) and the $H$. attenuata half cultured further. Figure $4 \mathrm{~A}$ indicates that $H$. oligactis desmonemes disappeared from the tentacles over a period of about 12 days in the absence of $H$. oligactis tissue.

The observed displacement of $H$. attenuata desmonemes (Fig. 3A) and its dependence on the presence of $H$. oligactis tissue in heterografts (Fig. 4A) is surprising in view of the well-known migration capacity of interstitial cells in Hydra tissue (Heimfeld and Bode, '84). Due to migration one would expect intestitial cells in both species to be homogeneously mixed after several days of parabiosis. Indeed, we could demonstrate extensive interstitial cell migration between $H$. oligactis and $H$. attenuata tissue in heterografts. In att/oli heterografts, in which $H$. oligactis was labeled with ${ }^{3} \mathrm{H}$-thymidine, $17 \%$ of the interstitial cells in the apical $H$. attenuata half were labeled 2 days after grafting.

reflects the relative numbers of $H$. oligactis/H. attenuata nematocytes produced between two time points. Each value represents the results from an single animal. $B$. Occurrence of $H$. attenuata desmonemes in tentacles of oli/att heterografts (same procedure as in A). 


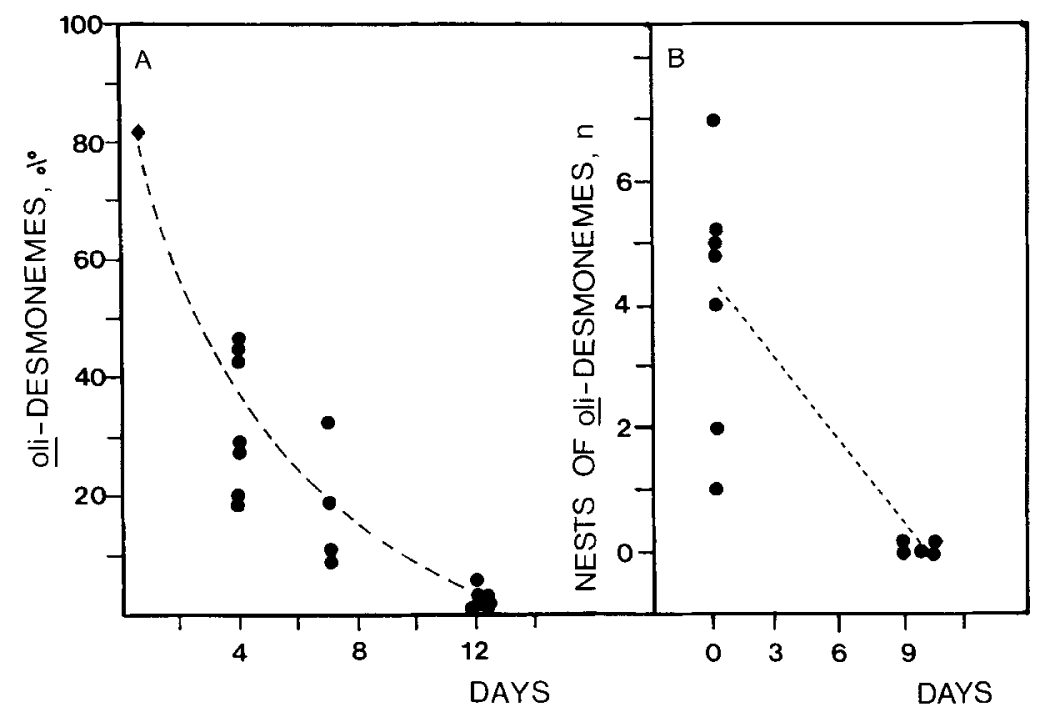

Fig, 4. Displacement of $H$. oligactis desmonemes from $H$. attenuata tissue. Each value represents the results from an single animal. A. After 10 days of parabiosis attoli heterografts were separated at the graft boundary, the apical $H$. attenuata portions were cultured, and the tentacles were analyzed for presence of $H$. oligactis

In oli/att heterografts, in which $H$. attenuata was labeled with ${ }^{3} \mathrm{H}$-thymidine, $9 \%$ of the interstitial cells in the apical in the apical (H. oligactis half were labeled 2 days after grafting. The extent of this interstitial cell migration is similar to that reported for $H$. attenuata/H. attenuata homografts (Heimfeld and Bode, '84).

To test whether migrating interstitial cells can differentiate nematocytes in the foreign tissue we used a histochemical procedure which specifically stains the nematocyst capsules of differentiating nematoblasts (David and Challoner, '74). The capsules of $H$. oligactis desmonemes and holotrichous isorhizas are clearly distinguishable from those of H. attenuata (Lee and Campbell, '79). Figure 5 shows a nest of $H$. oligactis desmonemes in the $H$. attenuata portion of a heterograft. An average of four such nests were found in the $H$. attenuata half of each heterograft beginning 2-3 days after grafting (Fig. 6). Nests of holotrichous isorhizas of the $H$. oligactis type were also observed although they were less frequent. In contrast, no $H$. attenuata desmoneme nematoblasts were found in the $H$. oligactis portion of oli/att heterografts (Fig. 6).

The migration of $H$. oligactis interstitial cells into $H$. attenuata tissue and the differ- desmonemes. Solid diamond, value from Figure 3A. B. After 2 days of parabiosis att/oli heterografts were separated at the graft boundary and the $H$. attenuata portions were cultured. These animals were stained with lead nitrate thioacetic acid on day 0 and day 8 to identify nests of differentiating $H$. oligactis desmonemes.

entiation capacity of $H$. oligactis nematoblasts in $H$. attenuata tissue suggest that a mixture of both $H$. attenuata and $H$. oligactis interstitial cells occurs in att/oli heterografts. To test the stability of this mixed population of interstitial cells we isolated the $H$. attenuata portion of heterografts, cultured them for several days, and stained them for differentiating nematoblasts. The results in Figure 4B indicate that nests of $H$. oligactis desmonemes disappear from isolated $H$. attenuata tissue within 9 days. Since both $H$. oligactis nematocytes (Fig. 4A) and $H$. oligactis nematoblasts (Fig. 4B) are displaced following removal of $H$. oligactis tissue, we conclude that mixed populations of $H$. oligactis and $H$. attenuata interstitial cells are not stable.

\section{Evidence for phagocytosis}

An active phagocytic system has long been known to be present in coelenterates (Metschnikoff, '05) and recent studies in Hydra have demonstrated phagocytosis by epithelial cells under certain conditions (Campbell, '76; Fujisawa and David, '84; Bosch and David, '84). Hence we investigated whether displacement of one species in heterografts was due to phagocytosis of that species by the other. Phagocytosis was estimated quantitatively by counting the number of epi- 


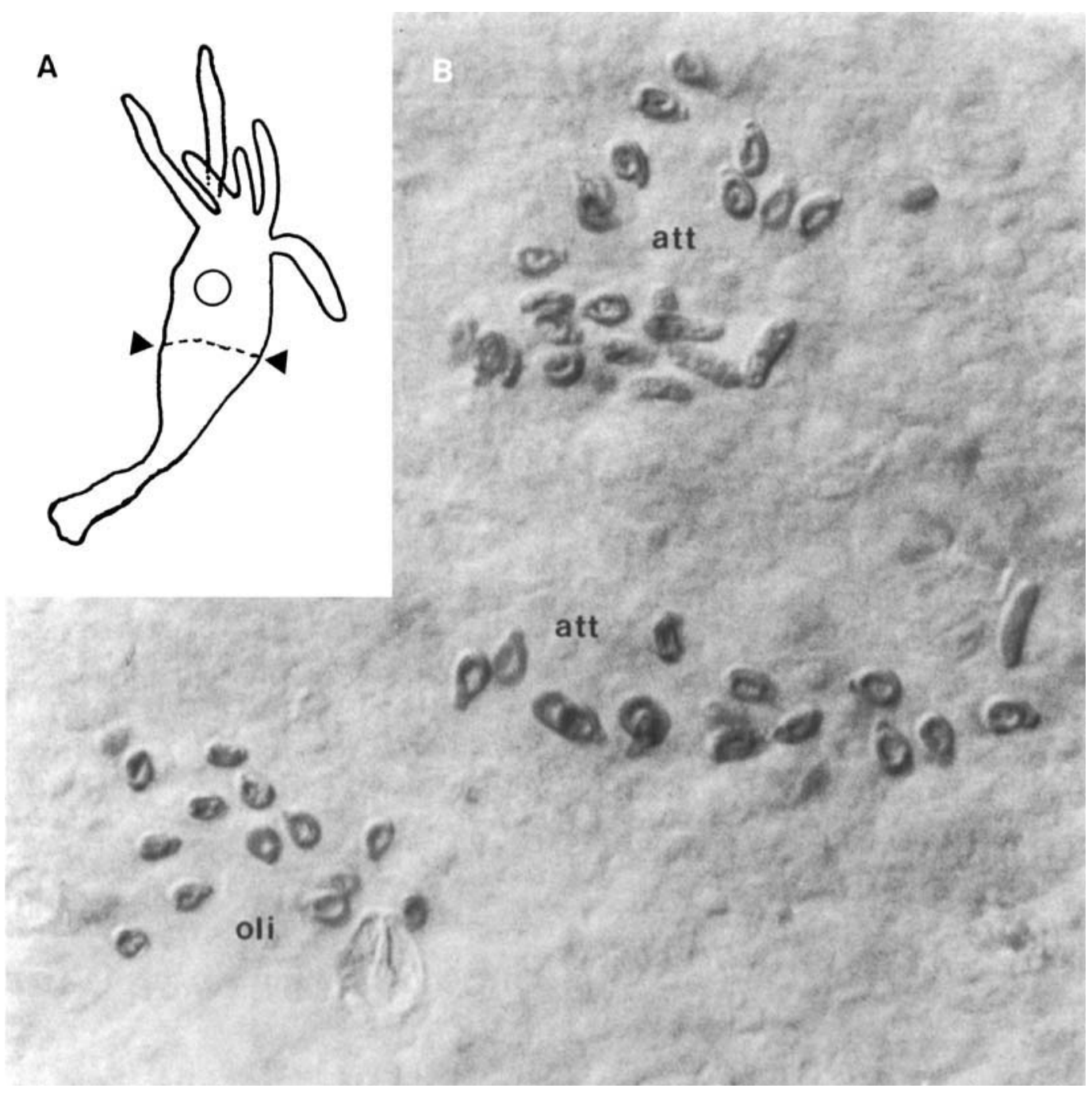

Fig. 5. Nests of differentiating desmoneme nematoblasts in the $H$. attenuata portion of an attoli heterograft stained with thioacetic acid and lead nitrate. A. Camera Iucida drawing of the heterograft detailed in B indicating the boundary between the two partners (arrow-

thelial cells containing phagocytic vacuoles in macerated, Feulgen-stained preparations of tissue from heterografts.

Figure 7a shows an epithelial cell containing a phagocytized cell in a vacuole. In heterografts two to three times more epithelial cells containing such phagocytic vacuoles were found at the graft site than in other body regions (Fig. 8). Such cells were found both in endoderm and ectoderm. By comparison, homografts between individuals of the same species did not contain more phagocy- heads) and the localization of the detailed view (circle). B. Detailed view of the $H$. attenuata region of an attoli heterograft 3 days after transplantation showing one nest of $H$. oligactis desmonemes and two nests of $H$. attenuata desmonemes. $\times 1,500$.

tized cells than ungrafted control animals. The somewhat enhanced levels of phagocytosis in the basal half of att/oli heterografts appear to be due to migration of $H$. attenuata interstitial cell into $H$. oligactis tissue and to the more effective phagocytosis of $H$. attenuata cells by $H$. oligactis (see Discussion).

Recognition and phagocytosis of foreign cells

To investigate whether phagocytosis involved cells of the opposite species, one partner in heterografts was prelabeled with ${ }^{3} \mathrm{H}$ - 


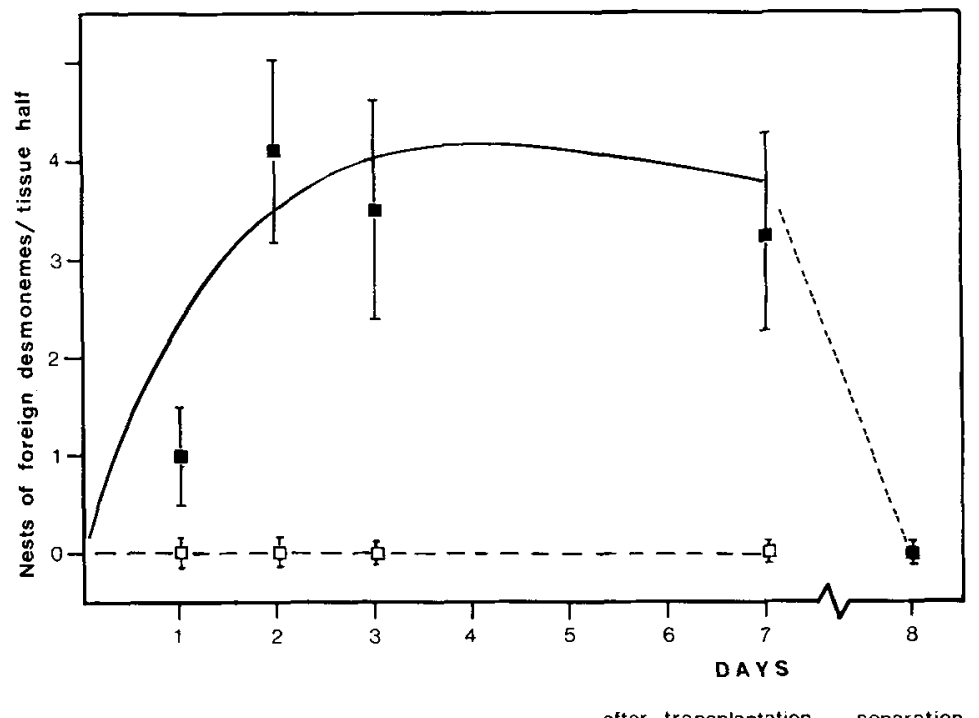

Fig. 6. Number of nests of differentiating desmoneme nematoblasts in heterografts stained with thioacetic acid and lead nitrate at various times after transplantation. Solid squares, nests of $H$. oligactis desmonemes in the
$H$. attenuata portion of att/oli heterografts. Hallow squares, nests of $H$. attenuata desmonemes in the the $H$. oligactis portion of oli/att heterografts. Symbols represent mean ( \pm S.D.) of five to seven animals.

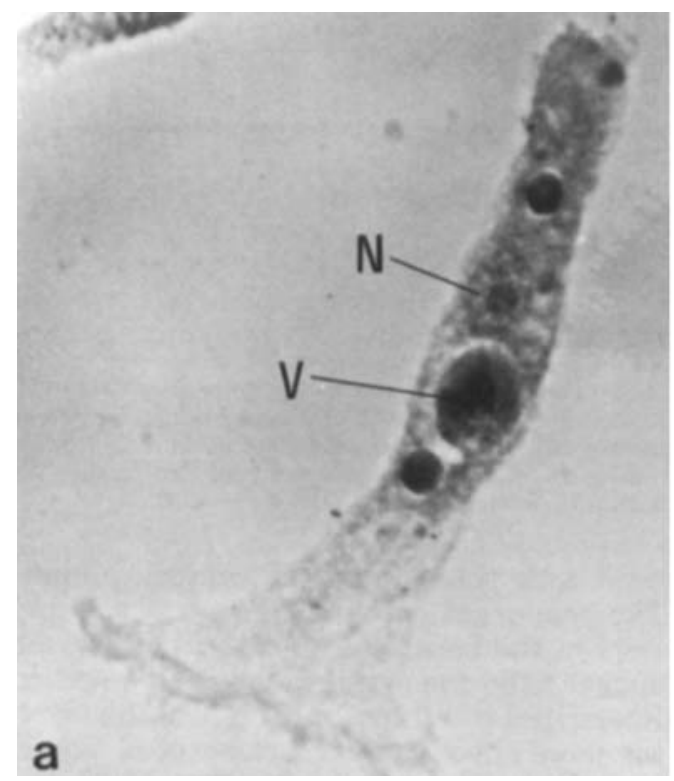

Fig. 7. a, Epithelial cell containing phagocytozed cells. Stained by the Feulgen method with Light Green counterstain $(V=$ vacuole, $N=$ nucleus $), \times 1,370$. Such phagocytic vacuoles were found in ectodermal and in endodermal epithelial cells at the graft region. They have been described in detail previously (Bosch and

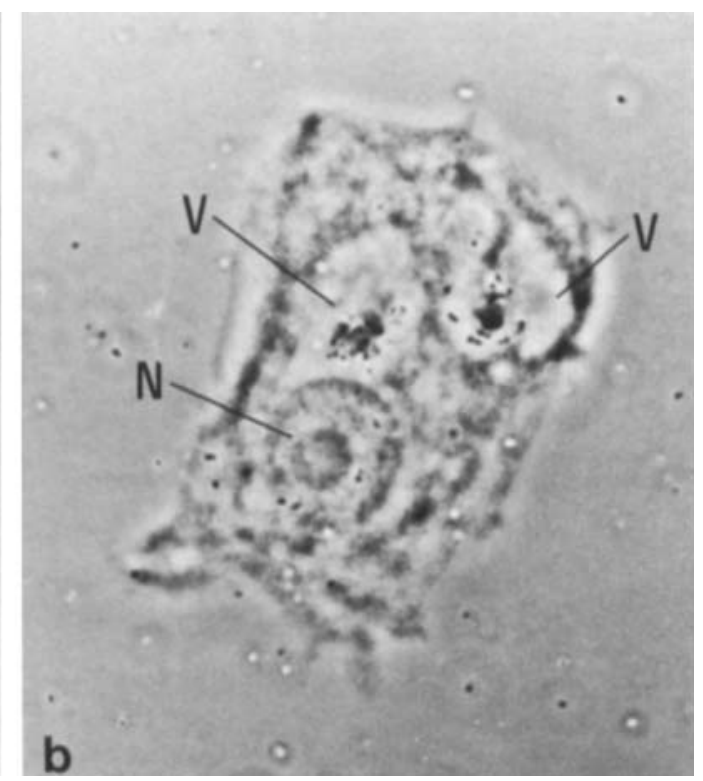

David, '84). b. Autoradiograph of an epithelial cell containing a ${ }^{3} \mathrm{H}$-thymidine-labeled vacuole $(\mathrm{V}=$ vacuole, $\mathrm{N}$ $=$ nucleus) $\times 2,370$. From the graft junction of an att/oli heterograft in which the $H$. attenuata partner was labeled with ${ }^{3} \mathrm{H}$-thymidine. 


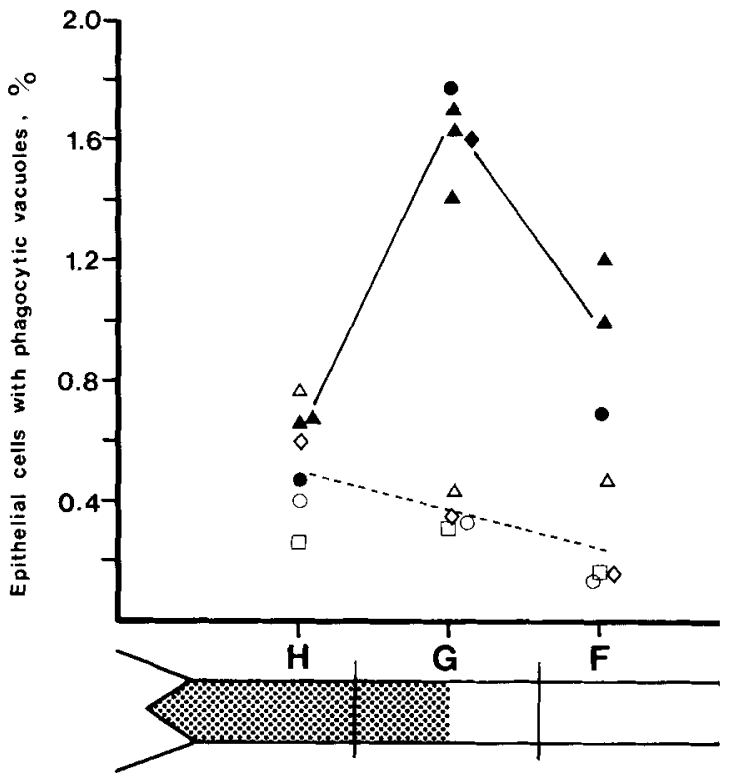

Fig. 8. Frequency of epithelial cells containing phagocytic vacuoles in different body regions of heterografts. After several days of parabiosis eight to twelve hetero grafts were cut in three parts $(H=$ head region, $G=$ graft region, $F=$ foot region), macerated, and stained by the Feulgen method with Light Green counterstain. The percentage of epithelial cells containing phagocytic vacuoles was scored. Heterografts used:

(๑), $H$. attenuata/H. oligactis 1 day after transplantation. $(\Delta), H$. attenuata/ $H$. oligactis 3 days after transplantation. (\$), $H$. attenuata/H. attenuata 3 days after transplantation. Controls: $(O), H$. attenuata/ $H$. attenuata 3 days after transplantation. $(\triangle), H$, oligactis $/ H$. oligactis 3 days after transplantation. ( $\square), H$. attenuata, $(\diamond), H$. oligactis, standard animals.

thymidine by repeated injections into the gastric cavity at 12-hour intervals for 2 days. This procedure labels about $80 \%$ of the epithelial cells and $100 \%$ of the interstitial cells. Apical halves of labeled $H$. attenuata or $H$. oligactis were then grafted to unlabeled proximal halves of the opposite species. After 3 days of parabiosis heterografts were cut into head, graft, and foot regions, macerated, and autoradiographed. Under these conditions 1$2 \%$ of epithelial cells in the graft region were observed to contain labeled vacuoles (Fig. 7b), indicating that cells of the opposite species were phagocytized. Labeled vacuoles were found irrespective of which partner was labeled in the heterografts. Hence, both $H$. attenuata and $H$. oligactis epithelial cells are capable of phagocytizing cells of the opposite species. Since cells are not phagocytized in homografts (Fig. 8) we conclude that epithelial cells are capable of recognizing nonself and reacting to it.

\section{Involvement of a memory component?}

In anthozoan cnidaria an enhanced response to second-set grafts has been reported in the scleractinian coral (Hildemann et al., '77) but not in gorgonian corals (Theodor, '70) nor in sea anemones (Lubbock, '80). In an attempt to demonstrate memory in the hydra immune response, we looked for an enhanced response to second-set heterografts. Primary grafts were separated after 2-4 days and the isolated partners cultured for further 2-6 days. Thereafter the same partners were grafted together again, cultured for several days, and the level of phagocytosis was measured. We found no evidence for a more vig. orous response (i.e., tissue rejection) nor an increase in the number of epithelial cells containing phagocytic vacuoles.

\section{DISCUSSION \\ Self/nonself recognition and the elimination of foreign cells}

Our results show that in grafts between $H$. attenuata and $H$. oligactis there is continuous displacement of $H$. attenuata epithelial cells (Fig. 1). Figure 2 demonstrates that displacement of $H$. attenuata by $H$. oligactis is not due to alterations in the rate of epithelial proliferation. The present study provides evidence that displacement is accompanied by phagocytosis of cells by both partners in heterografts (Fig. 7b). Since $H$. oligactis always displaces $H$. attenuata (Fig. 1) the simplest interpretation of these results is that $H$, oligactis is more efficient in phagocytizing $H$. attenuata than vice versa.

Displacement also affects cells of the interstitial cell linage in heterografts. Figures $4 \mathrm{~B}$ and 6 show that displacement of $H$. oligactis desmoneme nematoblasts occurs in the isolated $H$. attenuata half of att/oli heterografts. Displacement of $H$. attenuata desmoneme nematoblasts could not be directly demonstrated since such nematoblasts were not found in the $H$. oligactis portion of heterografts (Fig. 6). Nevertheless several results strongly support the idea that cells of the $H$. attenuata interstitial cell lineage are also displaced in heterografts. Our data show that a considerable number of $H$. attenuata interstitial cells $(9 \%)$ migrate into the $H$. oligactis half of oli/att heterografts. Thus it appears likely that our failure to observe differentiating $H$. attenuata desmonemes in the $H$. oligactis half of heterografts is due to the very effective phagocytosis of these cells by $H$. oligactis tissue. Such an explanation is consistent with the increased number of epithe- 
lial cells containing phagocytic vacuoles in the $H$. oligactis half of attoli heterografts (Fig. 8). Furthermore, in oli/att heterografts, in which $H$. attenuata was labeled with ${ }^{3} \mathrm{H}$ thymidine, epithelial cells containing labeled vacuoles were observed in the $H$. oligactis portion at positions well removed from the graft junction (data not shown). Such an increased rate of phagocytosis is probably also responsible for the absence of $H$. attenuata desmonemes in the tentacles of oli/att heterografts (Fig. 3B).

Thus, our results demonstrate that cells of both the epithelial and interstitial cell lineages are recognized and eliminated by nonself tissue. It seems likely that this recognition is mediated by cell-surface molecules and that cells of both lineages express these recognition signals. Such recognition signals are formally equivalent to the histocompatibility antigens present on cells of higher organisms.

\section{Previous studies concerning the compatibility of heterografts}

Our results confirm and extend previous examinations of att/oli heterografts. Issayev ('24), Goetsch ('24), and Kolenkine ('58, '71) all observed a gradual disappearance of $H$. attenuata tissue (epithelial cells) in grafts between $H$. attenuata and $H$. oligactis. In addition Goetsch ('24) demonstrated displacement of cells of the interstitial cell lineage based on identification of nematoblasts in whole mounts. Although displacement of $H$. attenuata nematocytes was complete in most cases, one graft appeared to contain some $H$. attenuata nematocytes 2 months after transplantation. Goetsch suggested that this animal might contain a stable mixture of interstitial cells of both species. Our results provide no evidence for a stable mixture of interstitial cells in heterografts (Figs. 3,4) and we view the one case reported by Goetsch ('24) as an example in which complete displacement of $H$. attenuata was delayed. Thus our results are in good agreement with previous observations of att/oli heterografts.

Recent observations of Lee and Campbell (79) on interstitial cell/epithelial cell chimeras of $H$. oligactis and $H$. attenuata do not appear to be consistent with our results. These authors repopulated epithelial $H$. attenuata with interstitial cells of $H$. oligactis. Although these chimeras could be maintained for up to six months they showed severe behavioral defects probably due to abnormal interstitial cell differentiation and to cell incompatibilities between the two species. The fact that $H$. oligactis interstitial cells were not displaced from these chimeras is surprising in view of our own results. Although we do not have an explanation it seems possible that $H$. attenuata interstitial cells or their products are necessary for displacement of $H$. oligactis cells and that therefore in epithelial $H$. attenuata no displacement can occur.

\section{Epithelial cells as effector cells of the immune response.}

Tissue recognition and histocompatibility reactions in hydra are mediated by epithelial cells (Fig. 7). The observation that epithelial cells are directly involved in the histocompatibility reaction is surprising since in other phyla specific phagocytic cells, rather than epithelial cells, are involved in immune responses. However, it should be noted that ectodermal as well as endodermal epithelial cells in hydra have previously been shown to be active phagocytes (Campbell, '76; Fujisawa and David, '84; Bosch and David, '84). Furthermore, recent work in a variety of organisms indicates that epithelial cells can phagocytose neighboring cells under certain conditions (e.g., hepatocytes, Bursch et al., '84). This process, which has been named "apoptosis" by Wyllie et al. ('80), appears to be widespread in both vertebrate and invertebrate organisms. Since phylogenetically old organisms like Hydra eliminate "nonself" by apoptosis, this kind of cell death may represent any evolutionary relic of an early immune response.

\section{Possible significance of self/nonself recognition in Hydra}

The role of self/nonself recognition in hydra is not immediately obvious since hydras exist as solitary polyps and are normally not confronted with foreign tissue as in our experimental situation. Hence it is interesting to ask what role such a recognition system may play in the natural habitat. Buss ('82) has suggested that systems of self/nonself recog. nition are required by organisms which proliferate asexually by budding ("ramet formation"). In such organisms one cell lineage must proliferate continuously during organismal growth and remain totipotent to form germ cells during sexual phases of the life cycle. Such organisms are susceptible to "infection" with variant cell lines which may 
compete with the totipotent host cell lineage for positions in the germline ("somatic cell parasitism"; Buss, '82; Buss and Green, '85). Thus an efficient self/nonself recognition system may have evolved in such organisms in order to combat overgrowth by somatic variants. Since gametes in Hydra arise from interstitial cells which proliferate continuously during growth and budding of the organism (Littlefield, '85; Bosch and David, in preparation), hydra would appear to require such a self/nonself recognition system in particular for the interstitial cell lineage. Our results demonstrate that just such a mechanism exists in hydra.

\section{ACKNOWLEDGMENTS}

We thank $P$. Tardent for sharing unpublished observations and T. Holstein for helpful discussion. The research was supported by the Deutsche Forschungsgemeinschaft (grant Da 163/1-1). T.C.G.B. is recipient of a postgraduate studentship of the University of Munich.

\section{LITERATURE CITED}

Bibb, C., and R.D. Campbell (1973) Cell affinity determining heterospecific graft tolerance in hydra. Tissue Cell, 5(2):199-208.

Bode, H, S. Berking, C.N. David, A. Gierer, H. Schaller, and E. Trenkner (1973) Quantitative analysis of cell types during growth and morphogenesis in hydra. Wilhelm Roux'Arch. Dev. Biol., 171:269-285.

Bosch, T.C.G., and C.N. David (1984) Growth regulation in Hydra: Relationship between epithelial cell cycle length and growth rate. Dev. Biol., 104:161-171.

Buehrer, M., and P. Tardent (1980) Compatibilities and incompatibilities in Podocoryne carnea (Anthomedusae). In: Developmental and Cellular Biology of Coelenterates. P. and R. Tardent, eds. Elsevier/NorthHolland Biomedical Press, Amsterdam, pp. 477-480.

Bursch, W., B. Lauer, I. Timmermann-Trosiener, G. Barthel, J. Schuppler, and R. Schulte-Herman (1984) Controlled cell death (apoptosis) of normal and putative preneoplastic cells in rat liver following withdrawal of tumor promotors. Carcinogenesis, 5:453-458.

Buss, L.W. (1982) Somatic cell parasitism and the evolution of somatic tissue compatibility. Proc. Natl. Acad. Sci. U.S.A., 79:5337-5341.

Buss, L.W., and D.R. Green (1986) Histoincompatibility in vertebrates: The relict hypothesis. J. Comp. Dev. Immunol., in press.

Buss, L.W., C.S. McFadden, and D.R. Keene (1984) Biology of hydractiniid hydroids. 2. Histocompatibility effector system/competitive mechanism mediated by nematocyt discharge. Biol. Bull., 167:139-158.

Buss, L.W., J.L. Moore, and D.R. Green (1985) Autoreactivity and self-tolerance in an invertebrate. Nature, 313:400-402.

Campbell, R.D., and C. Bibb (1970) Transplantation in coelenterates. Transplant. Proc., 2:202-211.

Campbell, R.D. (1976) Elimination of Hydra interstitial and nerve cells by means of colchizine. J. Cell Sci., 21:1-13.
Coombe, R.D., P.L. Ey, and C.R. Jenkin, (1984) Self/nonself recognition in invertebrates. Q. Rev. Biol. 59:231255.

David, C.N. (1973) A quantitative method for maceration of hydra tissue. Wilhelm Roux'Arch. Dev. Biol., 171:259-268.

David, C.N., and R.D. Campbell (1972) Cell cycle kinetics and development of Hydra attenuata. I. Epithelial cells. J. Cell Sci., 11:557-568.

David, C.N., and D. Challoner (1974) Distribution of interstitial cells and differentiating nematocytes in nests in Hydra attenuata. Am. Zool., 14:537-542.

Francis, L. (1973) Intraspecific aggression and its effect on the distribution of Anthopleura elegantissima and some related sea anemones. Biol. Bull, 144:73-92.

Fujisawa, T., and C.N. David (1984) Loss of differentiating nematocytes induced by regeneration and wound healing in Hydra. J. Cell Sci., 68:243-255.

Goetsch, W. (1924) Chimärenbildung bei Coelenteraten. Zool. Anz., 59:19-30.

Hauenschild, C. (1954) Genetische und entwicklungsphysiologische Untersuchungen über Intersexualität und Gewebeverträglichkeit bei Hydractinia echinata. Wilhelm Roux'Arch. Dev. Biol., 147:1-41.

Hauenschild, C. (1956) Uber die Vererbung einer Gewebeverträglichkeit bei dem Hydroid-polypen Hydractinia echinata. Z. Naturforsch., 11:132-138.

Heimfeld, S., and H.R. Bode (1984) Interstitial cell migration in Hydra attenuata. I. Quantitative description of cell movements. Dev. Biol., 105:1-9.

Hildemann, W.H. (1974) Some new concepts in immunological phylogeny. Nature, 250:116-120.

Hildemann, W.H. (1979) Immunocompetence and allogeneic polymorphism among invertebrates. Transplantation, 27:1-3.

Hildemann, W.H., R.L. Raison, G. Cheung, C.J. Hull, L. Akaka, and J. Okamoto (1977) Immunological specificity and memory in a scleractinian coral. Nature, 270:219-223.

Issayev, V. (1924) Researches on animal chimaeras. J. Genet., 14:273-349.

Kolenkine, X. (1958) Les modalités de l'association tissulaires après hétéro-greffe entre Hydra attenuata et Pelmatohydra oligactis. C.R. Acad. Sci. Paris, 246:16051608.

Kolenkine, X. (1971) Les histocompatibilités intraspécifique et interspécifique chez les hydres d'eau douce. Arch. Zool. Exp. Gen., 112:63-70.

Lackie, A.M. (1980) Invertebrate immunity. Parasitology, 80:393-412.

Lee, H., and R.D. Campbell (1979) Development and behavior of an intergeneric chimera of hydra (Pelmatohydra oligactis interstitial cells and Hydra attenuata epithelial cells). Biol. Bull, 157:288-296.

Littlefield, C.L. (1985) Germ cells in Hydra oligactis males: I. Isolation of a subpopulation of interstitial cells that is developmentally restricted to sperm production. Dev. Biol, 112:185-193.

Lubbock, R. (1980) Clone-specific cellular recognition in a sea anemone. Proc. Natl. Acad. Sci. U.S.A. 77:66676669.

Metschnikoff, E. (1905) Immunity in infective diseases. Cambridge Univ., London.

Müller, W. (1964) Experimentelle Untersuchungen über Stockenwicklung, Polypendifferenzierung und Sexualchimären bei Hydractinia echinata. Wilhelm Roux'Arch. Dev. Biol., 155:181-268.

Rubin, D.I., and H.R. Bode (1982) The aberrant, a morphological mutant of Hydra attenuata, has altered inhibition properties. Dev. Biol., 89:316-331.

Theodor, J.L. (1970) Distinction between "self" and "not- 
self" in lower invertebrates. Nature, 227:690-692.

Wanek, N., and R.D. Campbell (1982) Roles in ectodermal and entodermal epithelial cells in hydra morphogenesis: Construction of chimeric strains. J. Exp. Zool.,
221:37-47.

Wyllie, A.H., J.F.R. Kerr, and A.R. Currie (1980) Cell death: the significance of apoptosis. Int. Rev. Cytol,, 68:251-306. 